\title{
Homologous Core Collapse in a Massive Star and Self-Similar Evolution of Rebound Shocks
}

\author{
Yi Cao and Yu-Qing Lou
}

\author{
Department of Physics and Tsinghua Center for Astrophysics, Tsinghua University, Beijing, 100084, China
}

\begin{abstract}
During the gravitational core collapse of a massive progenitor star which may give rise to at least a class of gamma-ray bursts (GRBs) associated with supernovae, a stellar core rapidly passes through a short yet important phase of neutronization, producing a huge amount of energetic neutrinos and photons which contribute to the total pressure within the progenitor core. The collection of neutrinos, photons and gas materials together may be approximated as a fluid with a polytropic index $\gamma=4 / 3$ under the action of self-gravity. With a substantial generalization and using analytical and numerical methods (Lou \& Cao 2008), we recently constructed and examined various self-similar solutions to describe collapses, rebound shocks and flows systematically in a $\gamma=4 / 3$ polytropic gas mixture with spherical symmetry, and compare our results with those of Goldreich \& Weber (1980). It is also possible to construct central void solutions without or with shocks. Various features and characteristics of this nonlinear relativistically hot gas dynamics, including asymptotic and exact solutions, are presented. This more general polytropic model analysis provides the dynamic basis of understanding the evolution of rebound shocks in supernovae (SNs) and the results may be also utilized to benchmark hydrodynamic simulations.
\end{abstract}

Keywords: Gamma-Ray Bursts, Hydrodynamics, Shock Waves, Stars: Interior, Supernovae: General, Stars: Winds, Flows PACS: 95.30.Qd, 98.38.Ly, 95.10.Bt, 97.10.Me, 97.60.Bw

\section{INTRODUCTION}

Long gamma-ray bursts (GRBs) are generally thought to originate from demises and explosions of massive stars. They release the gravitational energy by drastic core collapses and shock outbursts, producing characteristic radiations in different energy bands, including gamma rays, $\mathrm{X}$-rays and energetic neutrinos. While the key mechanism of GRBs still remains mysterious, various observations offer valuable clues to these extremely explosive "cosmic fireworks". Especially since the discovery of the precise match in positions between GRB980425 and SN1998bw, several GRBs are clearly associated with at least one class of supernovae (Type Ibc) which are related to deaths of old massive stars. Three other such associations GRB030329 and SN2003dh, GRB031203 and SN2003lw, GRB060218 and SN2006aj together with a recent X-Ray Outburst (XRO)-SN connection between XRO 080109 and SN 2008D (e.g., Woosley \& Bloom 2006; Soderberg 2008) were reported within the last decade. For long GRBs, dynamics of stellar core collapses and rebound shock evolution in the context of SN explosions are important to both theories and observations of GRBs.

With certain assumptions about the state of collapsing stellar cores, hydrodynamics and magnetohydrodynamics (MHD) may be adopted to model these stellar collapses with or without shocks (e.g., Lou \& Wang 2006, 2007). Based on gas properties, a self-similar dynamics may emerge in these astrophysical processes. First, to simplify the problem, a quasi-spherical symmetry is presumed and only self-gravity and thermal pressure are taken into account in this semianalytic model of a relativistically hot polytropic gas. A simple yet useful assumption is the conventional polytropic state function, i.e., $P=\kappa \rho^{\gamma}$ where $P$ and $\rho$ are pressure and density, $\gamma$ is the polytropic index and $\kappa$ is a global constant. Based on such an assumption, valuable information about the stellar structure, solar physics, star formation and SN explosions may be obtained (e.g., Chandrasekhar 1939; Shu 1977; Lou \& Shen 2004; Suto \& Silk 1988; Lou \& Wang 2006, 2007). A simple argument on total energy of a static polytropic sphere demonstrates that $\gamma>4 / 3$ cases are stable while $\gamma<4 / 3$ ones are unstable (Chandrasekhar 1939). Thus most researchers focus on $1 \leq \gamma<4 / 3$ cases.

We emphasize that the critical case of $\gamma=4 / 3$ is important in astrophysical contexts. Statistical physics illustrates that in a temperature much lower than the Fermi energy, the state of particles whose rest mass is negligible is a $\gamma=4 / 3$ polytrope. Meanwhile, numerical simulations show that $\gamma=4 / 3$ polytrope is a sensible approximation for the central state of a compact object. In addition, the pressures of neutrinos and of photons, both crucial in collapsing stellar cores, may also be approximated by a $\gamma=4 / 3$ polytropic state. It turns out that the conventional polytropic assumption limits $\gamma=4 / 3$ situation severely in a self-similar model. In a classic paper on $\gamma=4 / 3$, Goldreich \& Weber (1980; GW hereafter) considered homologous collapse and concluded that when the pressure decreases by a fraction of no more than $2.9 \%$, a homologous core collapse would occur in the stellar interior, which is considerably 
lower than a value of $26 \%$ from numerical simulations of Bethe et al. (1979). GW tried to reduce this difference by introducing an inner core in a progenitor. Yahil (1983) performed a polytropic analysis and treated GW results as a limit of $\gamma \rightarrow(4 / 3)^{-}$.

In contrast to the conventional gas of a constant $\kappa$, a more general polytropic gas still obeys

$$
\frac{D}{D t} \log \left(\frac{P}{\rho^{\gamma}}\right)=\left(\frac{\partial}{\partial t}+\vec{u} \cdot \nabla\right) \log \left(\frac{P}{\rho^{\gamma}}\right)=0
$$

for the specific entropy conservation along streamlines with a variable $P / \rho^{\gamma}$ (e.g., Cheng 1977, 1978). Clearly, the conventional polytrope is only a special case. Nonetheless, mathematical derivations become more involved if the more general polytrope is adopted in the model analysis.

Recently, Lou \& Cao (2008) used the self-similar transformation (Fatuzzo et al. 2004) for a more general polytrope of $\gamma=4 / 3$ (see eqn (1)). Based on different values of a scaling index $a$, the problem is divided into three classes. Some essential procedures, analyses, and results are summarized in the following.

\section{MODEL FORMULATION AND MAIN RESULTS}

In spherical polar coordinates $(r, \theta, \phi)$ with spherical symmetry, physical variables are functions of radius $r$ and time $t$. Conservations of mass and momentum together with the local specific entropy conservation along streamlines govern the dynamic evolution of a relativistically hot gas of $\gamma=4 / 3$. Without shocks, the problem is invariant under the time reversal transformation. Thus, an expansion solution can also describe a collapse solution by such a transformation. In order to solve hydrodynamic partial differential equations (PDEs), a self-similar transformation is introduced to reduce PDEs to ordinary differential equations (ODEs), namely

$$
x=A t^{a} r, \quad \rho=\frac{\alpha(x)}{4 \pi G t^{2}}, \quad M=\frac{m(x)}{A^{3} G t^{3 a+2}}, \quad u=\frac{v(x)}{A t^{a+1}}, \quad P=\frac{p(x)}{4 \pi G A^{2} t^{2(a+2)}},
$$

where $\rho, M, u$ and $P$ are respectively density, enclosed mass, radial velocity and pressure, $a$ is a scaling index in such a transformation, $G$ is the gravitational constant and $A$ is a dimensional parameter to make the independent self-similar variable $x$ dimensionless. Then the governing hydrodynamic PDEs are reduced to nonlinear ODEs

$$
\begin{array}{r}
(a x+v) x^{2} \alpha=(3 a+2) m, \\
(a x+v) \frac{d v}{d x}+\frac{1}{\alpha} \frac{d p}{d x}=-\frac{m}{x^{2}}+(a+1) v, \\
(a x+v) \frac{d}{d x} \log \left(\frac{p}{\alpha \gamma}\right)=2(2+a-\gamma), \\
(a x+v) \frac{1}{\alpha} \frac{d \alpha}{d x}+\frac{d v}{d x}=2\left(1-\frac{v}{x}\right) .
\end{array}
$$

For considerations of dynamic processes in the core of a massive star and especially rebound shocks of SNe associated with GRBs, we set $\gamma=4 / 3$ to approximate the state of gas materials in the compact central core. Generally speaking, we require $a$ being less than zero. Importantly, equation (3) implies a division of all cases into three classes, depending on the value of $a$ being greater than, equal to or less than $-2 / 3$. In the following analysis, we deal with these classes separately and obtain respective results. Comparing to $\mathrm{GW}$, our analysis to the $a=-2 / 3$ class represents a substantial generalization.

The case of $a=-2 / 3$. For $a=-2 / 3$, the reduced specific entropy conservation (5) becomes automatically satisfied. Meanwhile from equations (3) - (6), one can readily derive the following two relations

$$
v=\frac{2}{3} x, \quad \frac{1}{x^{2}} \frac{d}{d x}\left(\frac{x^{2}}{\alpha} \frac{d p}{d x}\right)=-\alpha+\frac{2}{3} .
$$

It is taken that the pressure $P$ is proportional to $\rho^{4 / 3}$, and we need to know the proportional coefficient as a function of $(r, t)$ to complete the problem. Physically, $\log \left(P / \rho^{4 / 3}\right)$ is proportional to specific entropy $s(r, t)$ in a polytropic 
gas. Once this distribution $s(r, t)$ is known, the gas dynamics is determined. A more general case in self-similar transformation is to allow $p=g(x) \alpha^{4 / 3}$ where $g(x)$ is a function of $x$ to be specified for a self-similar flow. For a given $g(x)$, equation (7) can be solved with proper initial or boundary conditions. In general, velocity cannot diverge at large radii. Therefore a boundary condition to this problem is that an outer boundary of zero density should exist.

The first cut is to adopt a constant specific entropy $s(r, t)$ everywhere, i.e., $g(x)=1$, as GW did. We confirmed the main results of GW. An essential result is that the central density has a critical minimum below which the solution of equation (7) has no vanishing $\alpha$ at a finite radius. This critical value of $\alpha$ is $\sim 101.88$, consistent with GW work. In the context of a stellar collapse, GW result is somewhat limited in certain aspects. Take an old star in a relatively stationary state as described by the Lane-Emden equation (e.g., Chandrasekhar 1939). As the central nuclear burning becomes insufficient, the whole system rapidly evolves into a homologous collapsing or pre-collapsing phase. GW estimated the largest pressure reduction fraction (denoted by $r_{c}$ here) for a homologous collapse (prior to a rebound) and obtained a value of $\sim 2.9 \%$ under their assumption for a polytropic sphere of $\gamma=4 / 3$. In contrast, numerical simulations of Bethe et al. (1979) show that this value $r_{c}$ in supernovae could be substantially larger, about $26 \%$. To reconcile, GW proposed that there is an inner and smaller core which obeys their results as the pre-collapsing phase.

To generalize GW analysis for a homologous collapse, we allow a fairly arbitrary form of $g(x)$ in our model and thus accommodate a broad class of solutions for the density profile. Such a variable $s(r, t)$ may lead to a consistent result with numerical simulations (e.g., Bethe et al. 1979). In our numerical exploration, we choose $g(x)=1 /(1+\varepsilon x)$ with $\varepsilon$ being a coefficient to measure the range of $g(x)$ variation. According to our experiments, when $\varepsilon=0.1$, the pressure reduction $r_{c}$ can reach $\sim 10 \%$ and when $\varepsilon=0.3, r_{c}$ is $\sim 26 \%$ as given by Bethe et al. (1979). Given idealizations, these explorations do reveal a simple fact that a variable distribution of specific entropy $s(r, t)$ can be extremely important and we could model this effect in a self-similar manner.

The case of $a<-2 / 3$. In these cases, equations (3) and (5) lead to a new form of state function below

$$
p=C_{0} m^{q} \alpha^{\gamma}, \quad q \equiv \frac{2(2+a-\gamma)}{(3 a+2)},
$$

where $C_{0}$ is a constant. Most importantly, the variable specific entropy $\log \left(P / \rho^{\gamma}\right)$ is connected with the enclosed mass $m(x)$ in a power-law form. The conservation of mass guarantees the conservation of specific entropy. For $q=0$ and thus $a=\gamma-2$, we have a conventional polytrope; if we further require $\gamma=1$ and thus $a=-1$, we then have an isothermal gas (Shu 1977; Lou \& Shen 2004). For $\gamma=4 / 3$ under consideration, $q=2 / 3$, i.e., $p=C_{0} m^{2 / 3} \alpha^{4 / 3}$, which is a completely different family of problem in contrast to $P=\kappa \rho^{4 / 3}$. Note that for $\gamma \neq 4 / 3$ cases, the constant $C_{0}$ can be absorbed by readjusting the $A$ parameter in the transformation but for $\gamma=4 / 3$, constant $C_{0}$ is independent and characterizes the local sound speed.

It is easy to derive two coupled nonlinear ODEs for $v(x)$ and $\alpha(x)$ from equations (3) - (6). Through analysis, we obtain all kinds of relevant solutions both asymptotically and numerically, with two globally analytical solutions. Numerical simulations are needed to decide the paths of various self-similar evolution.

In our analysis, we compare with known results, finding more general counterparts of known situations, such as the central free-fall solution (Larson 1969; Penston 1969), the expansion wave collapse solution (EWCS; Shu 1977), envelope expansion with core collapse solutions (EECC; Lou \& Shen 2004) and quasi-static solutions (Lou \& Wang 2006, 2007). Furthermore, two new types of asymptotic solutions are also found. Corresponding physical meanings are discussed for possible processes in astrophysics. To enlarge the range of astrophysical applications, shock waves are also included. For shock conditions, mass, momentum and energy should be all conserved across a shock front. Numerical solutions connecting different types of asymptotic solutions are constructed in our model, with relevant physical scenarios outlined (see Lou \& Cao 2008 for details).

Most interestingly, we can construct central void solutions without or with shocks in the framework of hydrodynamics involving thermal pressure and self-gravity. In a background of Einstein de Sitter expanding universe, Fillmore \& Goldreich (1984a, b) considered a collection of collisionless particles with self-gravity. In the presence of certain kinds of perturbations, voids can be generated surrounding the center of such a flow of collisionless particles. Similarly in our model, although without an expanding background, thermal pressure also has a tendency of expansion and thus provides a driving mechanism to produce expanding voids under favorable conditions. Observationally, voids are ubiquitous in various astrophysical contexts, such as supernova remnants, molecular clouds, hot bubbles, superbubbles, planetary nebulae and galaxy clusters (see Hu \& Lou 2008 and Lou \& Hu 2008 for more details).

The case of $a>-2 / 3$. Since the enclosed mass cannot be negative, $a>-2 / 3$ requires $v>-a x$ in equation (3). However, as we cannot obtain any solution with an outer boundary of finite radius, velocity should converge as $x$ 
goes to infinity. However, these two requirements are incompatible with each other. The requirement of $v>-a x$ will certainly lead to a divergent velocity at large $x$. These cases are thus excluded in our model consideration.

\section{SUMMARY AND CONCLUSIONS}

Both analytic and numerical self-similar solutions have been explored for a more general polytropic gas with $\gamma=4 / 3$ (Lou \& Cao 2008; Wang \& Lou 2008). We have directly extended the classical analysis of GW for a conventional polytropic gas of spherical symmetry with $\kappa=1$, giving a plausible resolution to the discrepancy between theoretical calculations (GW) and numerical simulations (Bethe et al. 1979). We then study distributions of specific entropy in a certain system or infer these from other available information on the basis of our theoretical knowledge. Once such an entropy distribution is known, the self-similar dynamical evolution of a homologous core collapse can be calculated readily from our model. Another family of solutions are also discussed with a new state function of $P \propto M^{2 / 3} \rho^{4 / 3}$. In addition to counterparts of various previously know types of analytic and asymptotic solutions at $\gamma \neq 4 / 3$, we find some new ones and discuss their physical implications. In Lou \& Cao (2008), we attempt to analyze the self-similar dynamics of a $\gamma=4 / 3$ polytropic gas systematically and hope that it would provide valuable and useful clues to the study of compact objects and high-energy explosive phenomena, such as shock breakouts of SN explosions and thus GRBs.

There are several physical processes, such as nuclear reactions, radiation pressure, neutrino transportation, general relativity, rotation and magnetic field effects, that are not included in our preliminary model analysis. However, given approximations and idealizations of our model, we hope that the results of this theoretical hydrodynamic analysis catch certain essential features of large-scale shocks in a relativistically hot gas.

\section{ACKNOWLEDGMENTS}

This research has been supported in part by the National Natural Science Foundation of China (NSFC) grants 10373009 and 10533020 at Tsinghua University, by the SRFDP 20050003088, the Yangtze Endowment and the National Undergraduate Innovation Training Project from the Ministry of Education at Tsinghua University, and by Tsinghua Center for Astrophysics (THCA).

\section{REFERENCES}

1. H. A. Bethe, G. E. Brown, J. Applegate, and J. M. Lattimer, Nucl. Phys., A324, 487-533 (1979).

2. S. Chandrasekhar, 1939, An Introduction to the Study of Stellar Structure, Dover Publications, Inc., London.

3. A. F. Cheng, Astrophys. J., 213, 537-547 (1977).

4. A. F. Cheng, Astrophys. J., 221, 320-326 (1978).

5. M. Fatuzzo, F. C. Adams, and P. C. Myers, Astrophys. J., 615, 813-831 (2004)

6. P. Goldreich and S. V. Weber, Astrophys. J., 238, 991-997 (1980).

7. R.-Y. Hu and Y.-Q. Lou, Mon. Not. R. Astron. Soc., (2008arXiv0808.2090H) in press (2008).

8. R. B. Larson, Mon. Not. R. Astron. Soc., 145, 405-422 (1969).

9. Y.-Q. Lou and Y. Cao, Mon. Not. R. Astron. Soc., 384, 611-629 (2008).

10. Y.-Q. Lou and R.-Y. Hu, Mon. Not. R. Astron. Soc., submitted (2008).

11. Y.-Q. Lou and Y. Shen, Mon. Not. R. Astron. Soc., 348, 717-734 (2004).

12. Y.-Q. Lou and W.-G. Wang, Mon. Not. R. Astron. Soc., 372, 885-900 (2006).

13. Y.-Q. Lou and W.-G. Wang, Mon. Not. R. Astron. Soc., 378, L54-L58 (2007).

14. M. V. Penston, Mon. Not. R. Astron. Soc., 145, 457-485 (1969).

15. F. Shu, Astrophys. J., 214, 488-497 (1977).

16. A. M. Soderberg, et. al., Nature, 453, 469-474 (2008).

17. Y. Suto and J. Silk, Astropphys. J., 326, 527-538 (1988).

18. W.-G. Wang and Y.-Q. Lou, Astrophys. Space Sci., 311, 363-400 (2007).

19. W.-G. Wang and Y.-Q. Lou, Astrophys. Space Sci., 315, 135-156 (2008).

20. S. E. Woosley and J. S. Bloom, Annu. Rev. Astron. Astrophys., 44, 507-576 (2006).

21. A. Yahil, Astrophys. J., 265, 1047-1055 (1983). 